\title{
Correction to: Artificial noise-assisted physical layer security in D2D-enabled cellular networks
}

Yajun Chen ${ }^{1 *}$, Xinsheng $\mathrm{Ji}^{1,2,3}$, Kaizhi Huang ${ }^{1}$, Jing Yang ${ }^{1}$, Xin $\mathrm{Hu}^{1}$ and Yunjia Xu${ }^{1}$

\section{Correction}

In the original publication [1] the name of author Yajun Chen was spelled wrong. The original article was updated to rectify this error.

Incorrect version:

Yanjun Chen.

Correct version:

Yajun Chen.

\section{Author details}

${ }^{1}$ National Digital Switching System Engineering and Technological R\&D

Center, No.7, Jianxue Road, Zhengzhou 450002, China. ${ }^{2}$ National Mobile Communications Research Laboratory, Southeast University, No.2, Southeast University Road, Nanjing 211189, China. ${ }^{3}$ National Engineering Lab for Mobile Networking Security, No.10, Westtucheng Road, Beijing 100876, China.

Published online: 24 November 2017

\section{Reference}

1. Artificial noise-assisted physical layer security in D2D-enabled cellular networks EURASIP Journal on Wireless Communications and Networking 2017 2017:178 https://doi.org/10.1186/s13638-017-0969-1

\footnotetext{
*Correspondence: chenyajun_cool@126.com

${ }^{1}$ National Digital Switching System Engineering and Technological R\&D

Center, No.7, Jianxue Road, Zhengzhou 450002, China
} 